The Astrophysical Journal, 643:222-225, 2006 May 20

(C) 2006. The American Astronomical Society. All rights reserved. Printed in U.S.A.

\title{
A SIMPLE MODEL OF RADIATIVE EMISSION IN M87
}

\author{
E. Vitanza, V. Teresi, D. Molteni, and G. Gerardi \\ Dipartimento di Fisica e Tecnologie Relative, Università di Palermo, Viale delle Scienze, Palermo, 90128, Italy; vteresi@unipa.it \\ Received 2005 September 26; accepted 2006 January 26
}

\begin{abstract}
We present a simple physical model of the central source emission in the M87 galaxy. It is well known that the observed X-ray luminosity from this galactic nucleus is much lower than the predicted one, if a standard radiative efficiency is assumed. Up to now the main model invoked to explain such a luminosity was the ADAF (advectiondominated accretion flow) model. Our approach supposes only a simple axisymmetric adiabatic accretion with a low angular momentum together with the bremsstrahlung emission process in the accreting gas. With no other special hypothesis on the dynamics of the system, this model agrees well enough with the luminosity value measured by Chandra.
\end{abstract}

Subject headings: accretion, accretion disks — black hole physics — hydrodynamics

\section{INTRODUCTION}

M87 is a widely studied galaxy. Many authors have written about its globular clusters and nucleus, about the hypothesis of the existence of a supermassive black hole at its center, and recently about its jet from the nucleus (Jordán et al. 2004; Wilson \& Yang 2002). One of the most investigated problems on the physics of this galaxy is the problem of its luminosity, particularly the luminosity of its nucleus. By virtue of Chandra X-Ray Observatory observations an estimate of the X-ray nuclear luminosity is about $7 \times 10^{40} \mathrm{ergs} \mathrm{s}^{-1}$ (Di Matteo et al. 2003). Moreover, the data from Chandra itself allow determination of the temperature, density, and radial speed profiles of the interstellar medium emitting at X-ray frequencies inside the accretion radius of the central black hole. From these quantities it is easy to calculate the Bondi accretion rate, $\dot{M}_{\text {Bondi }}=0.1 M_{\odot} \mathrm{yr}^{-1}$ (Di Matteo et al. 2003). Supposing a canonical radiative efficiency $(\eta=0.1)$, the estimated luminosity is $5 \times 10^{44} \mathrm{ergs} \mathrm{s}^{-1}$, much higher than the measured value. A common way of solving this problem is based on the assumption of the ADAF model (advection-dominated accretion flow; Chen et al. 1997) for the accreting gas. In this model a significant part of the energy produced by viscosity is advected toward the central object, and therefore the fraction of gravitational energy transformed into emitted radiative energy is much lower than in the canonical Shakura-Sunyaev model (Shakura \& Sunyaev 1973). Moreover, to solve the energy excess problem, some authors have formed the hypothesis that another large part of the produced energy could be converted into the kinematic energy of the matter outflowing from the nucleus by the highly energetic jet (Wilson \& Yang 2002; Marshall et al. 2002).

In our approach, instead, we consider a steady state, axisymmetric (with a low angular momentum), and adiabatic accretion model. Such a model, by virtue of the low value of angular momentum, is a little refinement of the standard Bondi flow. The hypothesis of an influence of core rotation on the X-ray emission in a large, slowly rotating, elliptical galaxy was already suggested by Kley \& Mathews (1995). The emission process we suppose is the electron-ion thermal bremsstrahlung. This type of emission was already considered in the ADAF modeling of M87 (Ozel \& Di Matteo 2001), but not in the basic Bondi model framework. The nuclear X-ray luminosity calculated from our model is compatible with the observed value measured by Chandra. Although this model is very simple, it gives significant results with respect to the problem of the radiative emission modeling.

\section{THE PHYSICAL MODEL}

We set up a method to fit to the experimental data (the nuclear luminosity) using a model that is compatible with the known framework about the source (whose main ingredients are no evidence of high rotation, which implies quasi-spherical accretion, and bremsstrahlung emission process). Moreover, to perform such a kind of fit we need just one free parameter (the flow specific angular momentum), whereas the other variables are bound by the observed values at the accretion radius.

Since we consider only small angular momentum models, we can neglect the role of viscosity. Indeed, with the low angular momentum value we use, the gas will not rotate more than one orbit from the accretion radius to the black hole. In the presented model the rotational speed is so small that the gaseous structure is rather similar to a spherical one only slightly crushed in the vertical direction by the low angular momentum value. To obtain temperature, density, and radial speed profiles we consider a set of three equations, in which the symbols used have the following meanings: $\rho, v$, and $a$ are the density, gas radial speed, and sound speed, respectively, $H_{\text {disk }}$ is the half-thickness of the disk, $\dot{M}$ is the mass accretion rate, $\lambda$ is the specific angular momentum of the gas, $R_{g}$ is the Schwartzschild radius of the black hole, $\gamma=5 / 3$ is the ratio $c_{p} / c_{v}$ between the gas specific heats at constant pressure and volume, and $\rho_{\infty}$ and $a_{\infty}$ are the density and sound speed values, respectively, taken at a large distance from the black hole, in our case the values measured by Chandra at the black hole accretion radius.

With these definitions, the used equation system consists of the mass conservation equation,

$$
4 \pi r H_{\text {disk }} \rho v=\dot{M}=\text { constant, }
$$

the radial momentum equation,

$$
v \frac{d v}{d r}=-\frac{1}{\rho} \frac{d P}{d r}-\frac{G M}{\left(r-R_{g}\right)^{2}}+\frac{\lambda^{2}}{r^{3}},
$$

and the polytropic relation between density and sound speed,

$$
\rho=\rho_{\infty}\left(\frac{a}{a_{\infty}}\right)^{2 /(\gamma-1)}
$$


Equation (1) is the evaluation of the accretion rate $\dot{M}$ based on the idea that a mass flux $\rho v$ crosses a cylindrical surface of radius $r$ and height $2 H_{\text {disk, }}$ under the hypothesis that $\dot{M}$ is constant in space and time (steady accretion flow). Equation (2) is the radial momentum transfer equation, with the Lagrangian time derivative of the radial speed (without the term $\partial v / \partial t$, since we assume a steady state) in the first member and the three acting forces (pressure gradient, gravitational, and centrifugal) in the second one. Equation (3) is the thermodynamic relation between density and sound speed for an ideal gas during an adiabatic process.

The disk half-thickness is obtained through the following procedure. Using the hypothesis of vertical hydrostatic equilibrium, we can write

$$
\frac{1}{\rho} \frac{\partial P}{\partial z}=\frac{G M}{\left(r-R_{g}\right)^{2}} \frac{z}{r} .
$$

Although the flow we analyze cannot be described as a thin disk, the only way to evaluate the vertical height quantitatively is to make this assumption. This means that we approximate the vertical gradient $\partial P / \partial z$ with $P / H_{\text {disk }}$ and substitute the $z$-value in the right-hand side of equation (4) with $H_{\text {disk }}$ :

$$
\frac{1}{\rho} \frac{P}{H_{\mathrm{disk}}}=\frac{G M}{\left(r-R_{g}\right)^{2}} \frac{H_{\mathrm{disk}}}{r} .
$$

By some simple algebraic calculations, this leads to

$$
H_{\text {disk }}=\left(r-R_{g}\right) \sqrt{\frac{P}{\rho} \frac{r}{G M}} .
$$

We used the Paczyńsky-Wiita potential $V_{\mathrm{PW}}=-(G M) /\left(r-R_{g}\right)$ (Paczyńsky \& Wiita 1980) to mimic the general relativistic gravitational effects. Note that $v>0$ for inflowing gas. The pressure $P$ is given by $P=\rho a^{2} / \gamma$.

This scheme, containing one differential and two algebraic equations, can be substituted by a totally algebraic system of equations by using the Bernoulli relation instead of the radial momentum differential equation (2):

$$
\frac{v^{2}}{2}+\frac{a^{2}}{\gamma-1}-\frac{G M}{r-R_{g}}+\frac{\lambda^{2}}{2 r^{2}}=B
$$

where $B$ is the Bernoulli constant of the gas flow.

The algebraic equation system was solved using the following procedure. By introducing the Mach number $m=v / a$, solving equation (7) for $a$, and putting all the terms into relation (1), we obtained an equation in the unknown $m$,

$$
\dot{M}=-r m a K a^{2 /(\gamma-1)} \propto f(m) A(r, B, \lambda),
$$

where $K$ is a constant depending on the entropy of the system, $f$ is function only of the Mach number,

$$
f(m)=-\frac{m}{\left[m^{2} / 2+1 /(\gamma-1)\right]^{(\gamma+1) / 2(\gamma-1)}},
$$

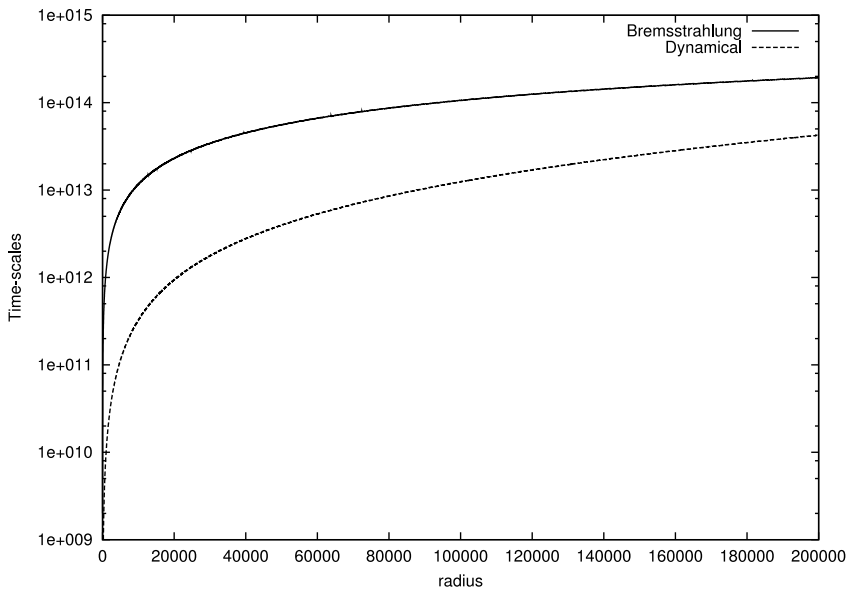

FIG. 1.-Comparison between the bremsstrahlung and the dynamical timescales vs. $r$. The times are in seconds.

and $A$ is function only of $r$ ( $B$ and $\lambda$ are parameters),

$$
A(r, B, \lambda)=r[B-V(r, \lambda)]^{(\gamma+1) / 2(\gamma-1)},
$$

with $V(r, \lambda)$, the effective body force potential (gravitational plus centrifugal), given by

$$
V(r, \lambda)=\frac{\lambda^{2}}{2 r^{2}}-\frac{G M}{r-R_{g}} .
$$

We used the values of density, flow radial speed, and sound speed $\rho_{\infty}, v_{\infty}$, and $a_{\infty}$, respectively, at the black hole accretion radius to calculate the Bernoulli constant $B$, necessary to solve the algebraic system. The unknowns are $\rho, v$, and $a$. By solving for these quantities, we obtained their radial profiles $\rho(r), v(r)$, and $a(r)$. Finally, from $a(r)$ we found the temperature profile $T(r)=\left(m_{\mathrm{H}} a^{2}\right) /\left(2 \gamma k_{\mathrm{B}}\right)$, where $m_{\mathrm{H}}$ is the proton mass and $k_{\mathrm{B}}$ is the Boltzmann constant. From the density $\rho$ and the temperature $T$ at a certain radius $r$ we calculated the emitted power density at the same $r$ for the bremsstrahlung emission process. Defining $e_{\mathrm{ff}}$ as the emitted power density, $n_{e}$ and $n_{i}$ as the electron and ion densities in the gas, respectively, $Z$ as the atomic number of the ions, $g_{B}$ as the Gaunt factor, $e$ and $m_{e}$ as the electron charge and mass, respectively, and $h$ as the Planck constant, the formula we used for bremsstrahlung is as follows:

$$
e_{\mathrm{ff}}=\left(\frac{2 \pi k_{\mathrm{B}}}{3 m_{e}}\right)^{1 / 2} \frac{2^{5} \pi e^{6}}{3 h m_{e} c^{3}} T^{1 / 2} n_{e} n_{i} Z^{2} g_{B} .
$$

The quantities $n_{e}$ and $n_{i}$ can be calculated from the density $\rho$ by assuming that the accreting gas is a hot plasma of fully ionized hydrogen. We adopted in our model the bremsstrahlung emission mechanism because, as already pointed out, the dominant emission process for the M87 X-ray nuclear luminosity is the thermal bremsstrahlung that yields a peak in the X-ray band (Reynolds et al. 1996). We cut off any emission when the temperature is larger than $2 \times 10^{9} \mathrm{~K}$. The main reason for the temperature cutoff is that beyond the indicated temperature limit the radiation frequency falls in the $\gamma$ band, and therefore the nuclear emission does not contribute to the X-ray luminosity. It is clear that the procedure we followed is valid from a physical point of view if the emission process we considered does not affect the 


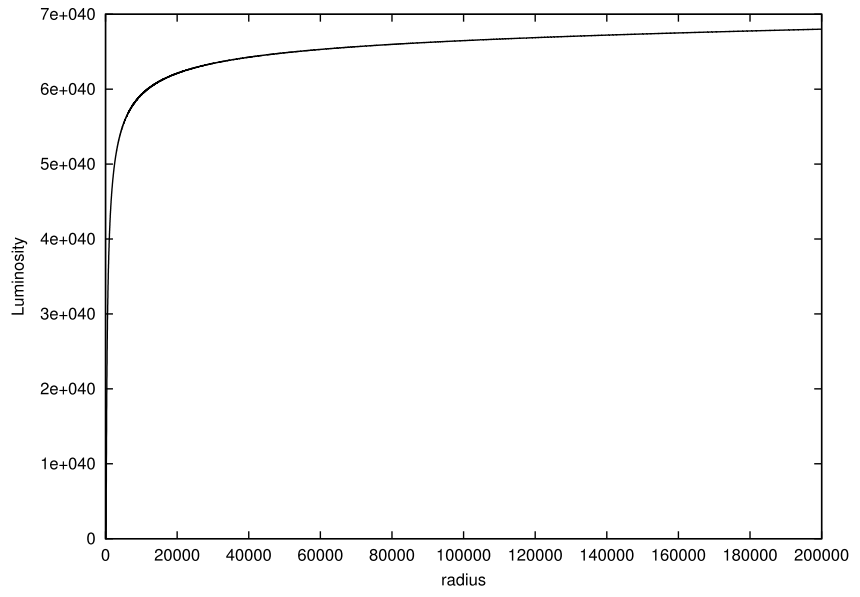

Fig. 2.-Partial luminosity $L(r)$ vs. $r$ in ergs per second.

flow structure very much (we did not include the corresponding terms in the energy equation). This means that the timescale of the process should be larger than the dynamical time of the flow. In $\S 3$ we show the comparison among these timescales.

We highlight that under the hypothesis of negligible viscosity, the algebraic method we followed is completely equivalent to the differential equation approach, since in this case the system is conservative (and the Bernoulli theorem eq. [7] holds). In particular, the algebraic method allows us to find the transonic flow with the sonic point at the same radius as in the differential equation approach. In the algebraic scheme the sonic point corresponds to a minimum of $A(r, B, \lambda)$ as a function of $r$ (for the mathematical details see the Appendix of Molteni et al. 1999), whereas in the differential equation approach the sonic point comes out from the regularity conditions on the function $v(r)$ (i.e., the usual conditions of the numerator and denominator of $d v / d r$ equal to zero). For conservative systems both methods give the same results concerning the sonic point position.

\section{RESULTS}

In this section we present the results obtained with a $\lambda$-value, measured in units of $c R_{g}$ (with $c$ the light speed and $R_{g}$ the Schwartzschild radius of the black hole), of 1.555 , which we found to be the value of the "best fit" of the calculated luminosity to the observed one. This value of angular momentum gives, at the black hole accretion radius, a rotational speed of

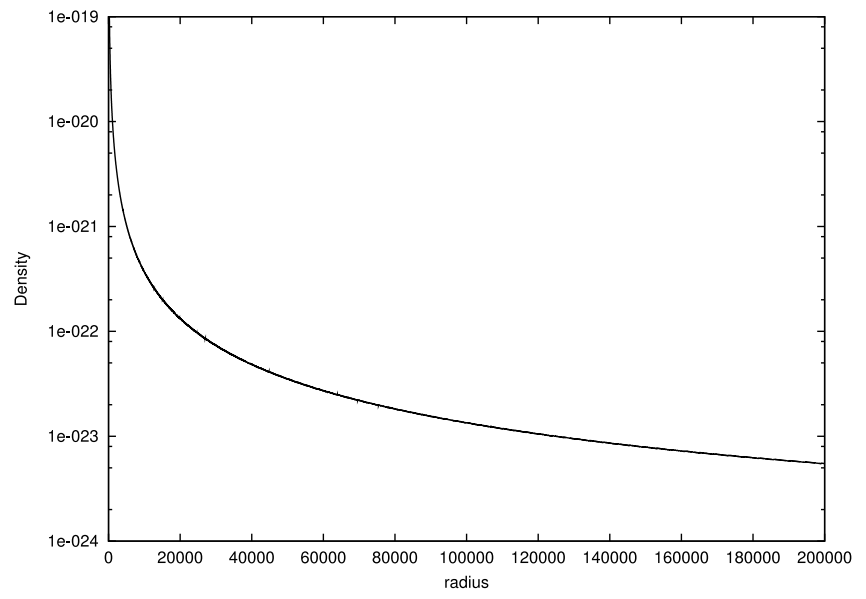

FIG. 3.-Density radial profile in grams per cubic centimeter.

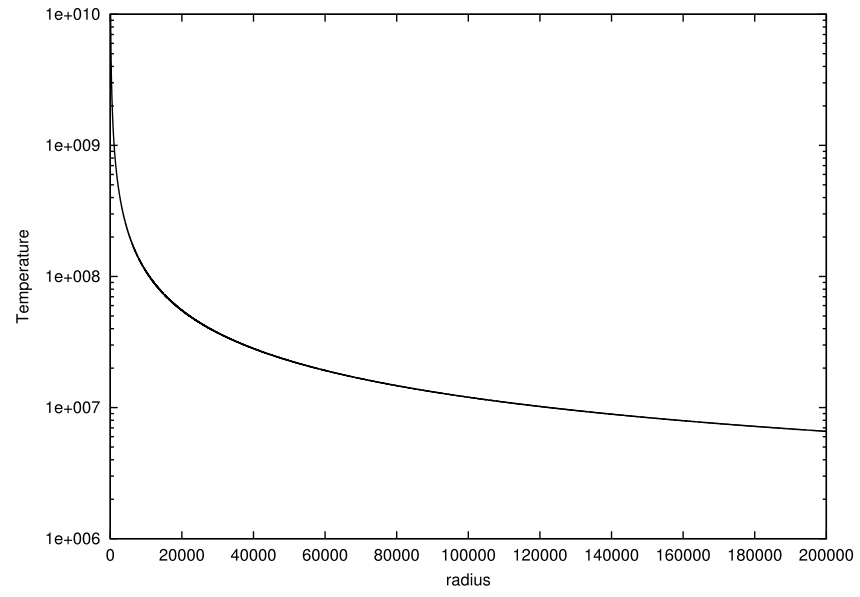

FIG. 4.-Temperature radial profile in kelvins.

$0.93 \mathrm{~km} \mathrm{~s}^{-1}$, which is within the observational error of the measured speed data (Cohen \& Ryzhov 1997). It is worth noting that by lowering $\lambda$, the flow structure given by our model gets closer and closer to the Bondi configuration until, when $\lambda=0$, it reaches approximately the Bondi model structure. All the data shown in this section concern the range $3 R_{g}<r<2 \times 10^{5} R_{g}$, since, according to our results, it is within this region that about $98 \%$ of the total luminosity is produced. As we already pointed out in $\S 2$, a criterion to assess the validity of our model in presence of radiative emission processes is that the flow is fundamentally adiabatic. This is verified if the emission timescale for each considered process is larger than the flow dynamical time $t_{\text {dyn }}=r / v$ at the same radius. We present in Figure 1 the values of these typical times at different radii in the range $3 R_{g}<r<2 \times 10^{5} R_{g}$. From this figure it is clear that the bremsstrahlung emission timescale is larger than the flow dynamical time. We do not consider the synchrotron emission because its frequency range falls in the radio and (via Comptonization) optical bands (Reynolds et al. 1996). As regards the soft X-ray emission lines, they have a significant intensity at low temperatures (about $10^{6} \mathrm{~K}$ ), which can be found only beyond the accretion radius.

Our model allows calculation of the luminosity emitted by the whole flow. We present in Figure 2 the partial luminosity $L(r)$ emitted from $3 R_{g}$ to a generic radius $r$. The figure shows that the largest part of the total luminosity emitted by the entire flow is produced in the region from $r=3 R_{g}$ to $2 \times 10^{5} R_{g}$. The

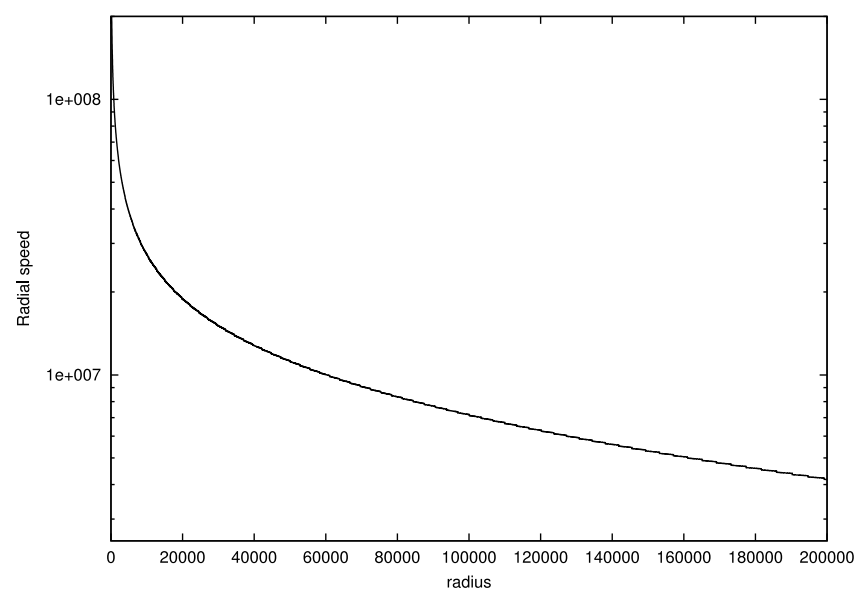

Fig. 5. - Flow radial speed vs. $r$ in centimeters per second. 


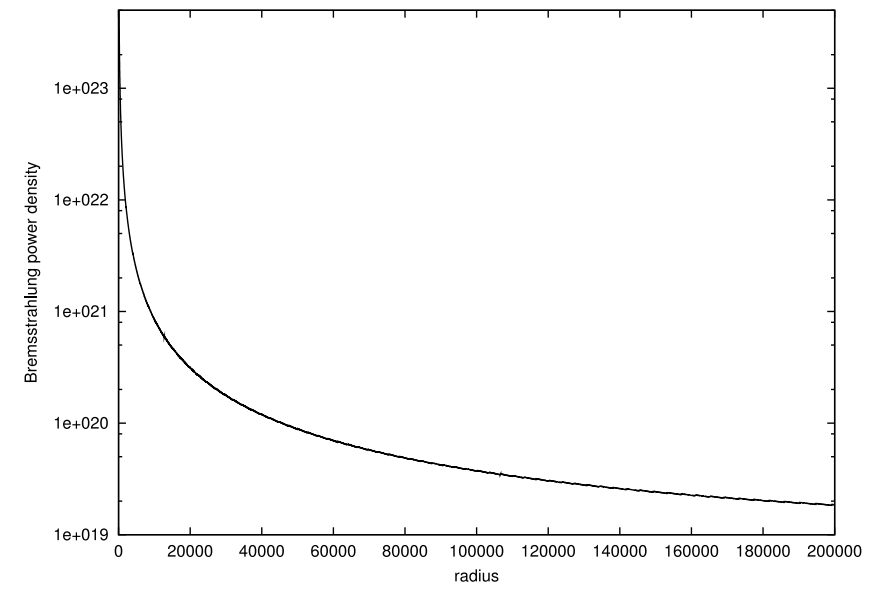

FIG. 6.-Radial density of the emitted power for the bremsstrahlung process. The power density is in ergs per centimeter per second.

luminosity coming out from the whole system (up to the external boundary at $r=5 \times 10^{5} R_{g}$ ) is $7.1 \times 10^{40} \mathrm{ergs} \mathrm{s}^{-1}$. Therefore, our model permits explanation of the observed luminosity as a result of the accretion flow emission. Obviously this picture is not the only possible one. For example, Wilson \& Yang (2002) attribute the origin of the nuclear X-ray emission to the parsec- or subparsec-scale jet. However, our hypothesis has the advantage of explaining the observational data in the simple framework of the interstellar medium accreting onto the central black hole.
In Figures 3, 4, and 5 we show the radial profiles of the three variables that characterize the flow structure: density, temperature, and radial speed. In Figure 6 we show the radial density of the emitted power for the bremsstrahlung process.

\section{CONCLUSIONS}

In this work we show that the addition of a small gas angular momentum to a simple adiabatic accretion flow together with the thermal bremsstrahlung emission process can give, for the active nucleus of the galaxy M87, a luminosity value that is in good agreement with the measured one, whereas the value obtained supposing the standard radiative efficiency is 4 orders of magnitude larger than the measured luminosity. We obtain this result using a very simple model that contains a new free parameter, the specific angular momentum $\lambda$ of the accreting gas, which can be adjusted in order to fit the model to the observed luminosity. With $\lambda=1.555$ the obtained luminosity value is $7.1 \times 10^{40} \mathrm{ergs} \mathrm{s}^{-1}$ versus a measured one of about $7 \times 10^{40} \mathrm{ergs} \mathrm{s}^{-1}$. Our result can also be considered as a way of giving an estimate of the gas angular momentum in the nucleus of M87. Moreover, our model could be applied to other sources in which the low observed luminosity requires a low radiative efficiency model.

We thank the anonymous referee for his suggestions, which helped us to improve the paper and make it more clear in the explanation of the astrophysical content.
Chen, X., Abramowicz, M. A., \& Lasota, J.-P. 1997, ApJ, 476, 61

Cohen, J. G., \& Ryzhov, A. 1997, ApJ, 486, 230

Di Matteo, T., Allen, S. W., Fabian, A. C., Wilson, A. S., \& Young, A. J. 2003, ApJ, 582, 133

Jordán, A., et al. 2004, ApJ, 613, 279

Kley, W., \& Mathews, W. G. 1995, ApJ, 438, 100

Marshall, H. L., Miller, B. P., Davis, D. S., Perlman, E. S., Wise, M., Canizares,

C. R., \& Harris, D. E. 2002, ApJ, 564, 683

\section{REFERENCES}

Molteni, D., Toth, G., \& Kuznetsov, O. A. 1999, ApJ, 516, 411

Ozel, F., \& Di Matteo, T. 2001, ApJ, 548, 213

Paczyńsky, B., \& Wiita, P. J. 1980, A\&A, 88, 23

Reynolds, C. S., Di Matteo, T., Fabian, A. C., Hwang, U., \& Canizares, C. R. 1996, MNRAS, 283, L111

Shakura, N. I., \& Sunyaev, R. A. 1973, A\&A, 24, 337

Wilson, A. S., \& Yang, Y. 2002, ApJ, 568, 133 